\title{
Src drives the Warburg effect and therapy resistance by inactivating pyruvate dehydrogenase through tyrosine-289 phosphorylation
}

\author{
Yue Jin ${ }^{1,2, *}$, Qingsong Cai ${ }^{1, *}$, Anitha K. Shenoy ${ }^{1}$, Sangbin Lim ${ }^{3}$, Ying Zhang ${ }^{1}$, Steve \\ Charles $^{1}$, Miriam Tarrash ${ }^{1}$, Xueqi Fu ${ }^{2}$, Sushama Kamarajugadda ${ }^{1}$, Jose G. Trevino ${ }^{4}$, \\ Ming $\operatorname{Tan}^{3}$, Jianrong Lu ${ }^{1}$ \\ ${ }^{1}$ Department of Biochemistry and Molecular Biology, UF Health Cancer Center, University of Florida College of Medicine, \\ Gainesville, FL 32610, USA \\ ${ }^{2}$ School of Life Sciences, Jilin University, Changchun 130012, China \\ ${ }^{3}$ Mitchell Cancer Institute, University of South Alabama, Mobile, AL 36604, USA \\ ${ }^{4}$ Department of Surgery, UF Health Cancer Center, University of Florida College of Medicine, Gainesville, FL 32610, USA \\ *These authors have contributed equally to this work \\ Correspondence to: Jianrong Lu, e-mail: jrlu@ufl.edu \\ Keywords: Warburg effect, pyruvate dehydrogenase, Src, reactive oxygen species, chemoresistance \\ Received: September 07, $2015 \quad$ Accepted: January 23, $2016 \quad$ Published: February 03, 2016
}

\section{ABSTRACT}

The Warburg effect, which reflects cancer cells' preference for aerobic glycolysis over glucose oxidation, contributes to tumor growth, progression and therapy resistance. The restraint on pyruvate flux into mitochondrial oxidative metabolism in cancer cells is in part attributed to the inhibition of pyruvate dehydrogenase (PDH) complex. Src is a prominent oncogenic non-receptor tyrosine kinase that promotes cancer cell proliferation, invasion, metastasis and resistance to conventional and targeted therapies. However, the potential role of Src in tumor metabolism remained unclear. Here we report that activation of Src attenuated PDH activity and generation of reactive oxygen species (ROS). Conversely, Src inhibitors activated PDH and increased cellular ROS levels. Src inactivated PDH through direct phosphorylation of tyrosine-289 of PDH E1a subunit (PDHA1). Indeed, Src was the main kinase responsible for PDHA1 tyrosine phosphorylation in cancer cells. Expression of a tyrosine-289 non-phosphorable PDHA1 mutant in Src-hyperactivated cancer cells restored PDH activity, increased mitochondrial respiration and oxidative stress, decreased experimental metastasis, and sensitized cancer cells to pro-oxidant treatment. The results suggest that Src contributes to the Warburg phenotype by inactivating PDH through tyrosine phosphorylation, and the metabolic effect of Src is essential for Src-driven malignancy and therapy resistance. Combination therapies consisting of both Src inhibitors and pro-oxidants may improve anticancer efficacy.

\section{INTRODUCTION}

Cancer cells typically exhibit enhanced glucose uptake, glycolysis, and lactate production regardless of oxygen availability, a phenotype known as aerobic glycolysis or the Warburg effect. Increased yield of intermediate glycolytic metabolites fuels macromolecule biosynthesis, thereby providing essential anabolic support to sustain cell proliferation and tumor growth $[1,2]$. The end product of glycolysis, pyruvate, is mostly kept away from mitochondrial oxidative metabolism in cancer. Therefore, in cancer cells, mitochondrial oxidation of pyruvate is uncoupled from glycolysis. Because mitochondrial respiration is the major cellular source of reactive oxygen species (ROS) [3], the Warburg metabolic phenotype allows cancer cells to avoid generating excess mitochondrial ROS from pyruvate oxidation, limit cellular oxidative stress, and thus acquire improved metastatic potential $[4,5]$. As conventional radiation and chemotherapy kill cancer cells in large part through 
generation of ROS [6], limiting ROS production also renders cancer cells less susceptible to oxidative stressinduced cell death and hence confers resistance to prooxidant therapy $[7,8]$. Overall, the Warburg effect promotes tumor growth, metastasis and therapy resistance.

Entry of glycolysis-derived pyruvate into mitochondrial oxidative metabolism is primarily governed by the pyruvate dehydrogenase complex (PDC) in mitochondria. PDC converts pyruvate to acetyl-CoA, which subsequently enters the tricarboxylic acid (TCA) cycle. Therefore, PDC is the gatekeeper enzyme that strategically links glycolysis to mitochondrial oxidation [9]. PDC has a profound impact on whether pyruvate should be oxidized in mitochondria or converted to lactate in cytosol, thereby critically controlling glucose metabolism and oxidative stress. Accumulating evidence suggests that inhibition of PDC is an important contributor to the Warburg effect in cancer cells. According to the current dogma, PDC is principally inactivated by phosphorylation of three specific serine residues on pyruvate dehydrogenase (PDH), the first and most important enzyme component of PDC $[9,10]$. Such phosphorylation is catalyzed by pyruvate dehydrogenase kinases (PDKs) [10]. Hypoxia-inducible factors (HIFs), oncoprotein Myc and canonical Wnt signaling can transcriptionally upregulate PDK expression, thereby attenuating PDC activity, pyruvate oxidation and ROS production [11-15]. Consistent with frequent activation of HIF, Myc and Wnt signaling in cancer, PDKs are overexpressed in a variety of human malignancies and contribute to the Warburg effect $[5,16,17]$.

While metabolic alterations are common in cancer, the underlying driving forces remain incompletely understood. Only a few oncogenes and tumor suppressors have been shown to rewire cellular metabolism [18, 19]. Src, a nonreceptor tyrosine kinase, is the most widely known and the prototypical member of the Src family of tyrosine kinases (SFKs) [20,21]. SFKs critically transmit signals downstream of cell surface receptors. Many Src family members have been identified as proto-oncogenes, and viral Src is indeed the first identified oncogene [22]. Aberrantly activated SFKs drive a multitude of malignant properties, including cell proliferation, survival, migration, invasion, angiogenesis and metastasis [23-25]. Src also confers resistance to traditional ionizing radiation and chemotherapy [26], as well as to endocrine and anti-HER2 targeted therapies in breast cancer [23], although the underlying mechanisms remain poorly understood. Src overexpression and/or hyperactivation are evident in a wide range of human cancers, and correlate with disease recurrence and adverse prognosis [21,23].

However, the key Src-dependent downstream signaling events contributing to the aggressive and therapy-resistant malignant phenotypes are not clearly elucidated. It was unclear whether and how Src might reprogram metabolism to foster malignancy. Here we found that Src directly phosphorylated PDH and was the major kinase responsible for PDH tyrosine phosphorylation in cancer cells. Tyrosine phosphorylation of PDH suppressed PDH activity, mitochondrial respiration and ROS generation, and increased metastasis and resistance to chemotherapy. The study thus identified a metabolic basis of Src's pro-malignant function.

\section{RESULTS}

\section{Activation of Src attenuates PDH activity and oxidative stress}

Cancer cells may acquire increased metastatic potential by attenuating $\mathrm{PDH}$ and mitochondrial oxidative stress [4]. Given Src's pro-metastatic role, we asked whether Src might modulate PDH activity and mitochondrial metabolism. To activate Src, we ectopically expressed Src527, a constitutively active form of Src [27, 28], in MCF10A untransformed mammary epithelial cells through lentiviral transduction. We prepared mitochondrial extracts from control and Src527expressing cells and measured PDH activity. MCF10A cells expressing activated Src displayed lower PDH activity than control cells (Figure 1A). PDH promotes pyruvate flux into mitochondrial oxidative metabolism, which is a major source of ROS [3]. As expected, Srcactivated MCF10A cells showed decreased ROS levels compared with control cells (Figure 1B). SW480 colon cancer cells displayed little intrinsic Src activity [29]. Similar to MCF10A cells, expression of Src527 in SW480
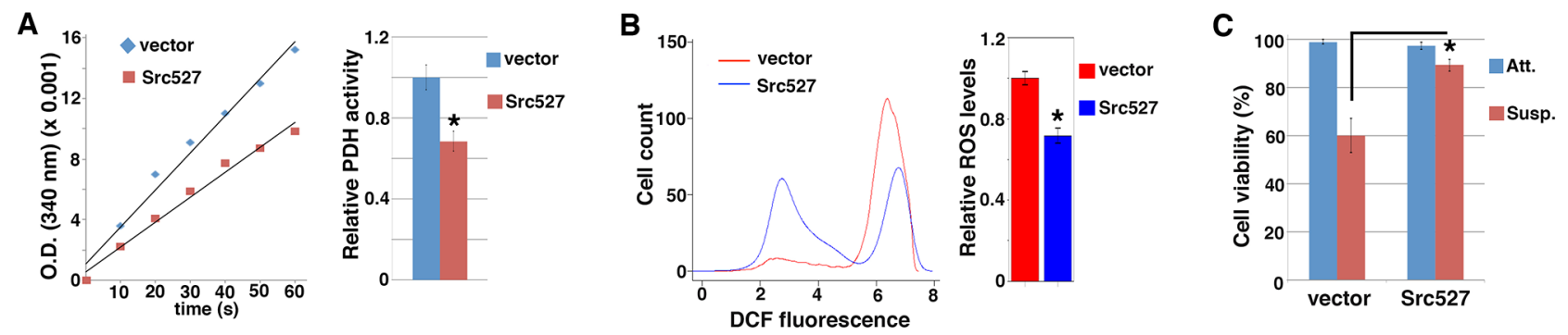

Figure 1: Activation of Src inhibits PDH activity and ROS generation, and confers anoikis resistance. MCF10A cells were transduced with lentivirus expressing activated Src527, and subjected to measurement of PDH activity A. ROS content B. and anoikis sensitivity C. Error bars represent standard deviation (S.D.). ${ }^{*} \mathrm{p}<0.05$. 
cells also resulted in suppression of PDH activity and ROS content (Supplementary Figure S1). Epithelial cells normally depend on attachment to extracellular matrix for growth and survival, and matrix detachment causes a form of apoptosis known as anoikis [30]. Anoikis is attributed to ROS, which arise from cell detachment [4, 31-34]. Consistently, Src-activated MCF10A cells survived in suspension significantly better than control cells (Figure 1C). Collectively, these results suggest that activation of Src inhibits PDH activity and ROS generation, and confers anoikis resistance.

Conversely, we tested whether inhibition of Src in cancer cells with heightened Src activity might activate PDH and enhance ROS production. Both 4T1 mammary carcinoma cells and AsPC1 pancreatic cancer cells showed hyper-activation of $\operatorname{Src}[35,36]$. Treatment of these cells with small molecule compounds SU6656 and Saracatinib [37-39], which are selective Src kinase inhibitors, markedly increased PDH activity and ROS levels (Figure 2A-2D). The observations confirm that Src suppresses $\mathrm{PDH}$ function and ROS production.

\section{PDH is tyrosine-phosphorylated in cancer cells in a Src-dependent manner}

PDKs inactivate PDH through serine phosphorylation of its E1 $\alpha$ subunit (PDHA1) [9, 10]. PDH is predominantly localized in mitochondria. Interestingly, Src family tyrosine kinases such as Src, Lyn, Fyn, and Fgr are also present in or transported into mitochondria as reported by multiple independent laboratories [40-47]. Therefore, we wondered whether Src might inhibit PDH activity through direct tyrosine phosphorylation of PDHA1.
To facilitate the detection of PDHA1 tyrosine phosphorylation, we generated a lentiviral vector expressing PDHA1 with a Flag tag on its carboxyl terminus (PDHA1-Flag). Following transfection into HEK293 cells, PDHA1-Flag proteins were immunoprecipitated with antiFlag antibodies and immunoblotted with phosphotyrosinespecific antibodies. When expressed alone in HEK293 cells, PDHA1-Flag was not tyrosine-phosphorylated (Figure 3A). However, when co-transfected with constitutively activated Src (Src527), PDHA1 showed strong tyrosine phosphorylation (Figure 3A). By contrast, a kinase-inactive Src295 mutant [48] failed to induce PDHA1 tyrosine phosphorylation, suggesting that Src causes PDHA1 tyrosine phosphorylation through its kinase activity.

Besides Src kinases [40-47], several receptor tyrosine kinases such as EGFR [42], HER2 [49] and FGFR1 [50] have been reported to be located in mitochondria. When co-transfected with PDHA1-Flag, wild-type (WT) and a constitutively active V659E mutant of HER2 [51] as well as EGFR did not lead to PDHA1 tyrosine phosphorylation (Figure 3A). FGFR1 was recently reported to phosphorylate PDHA1 [52]. FGFR1 induced weakly detectable tyrosine phosphorylation of PDHA1 in this assay (Figure 3A).

Based on Src's ability to induce PDHA1 tyrosine phosphorylation, we verified whether PDHA1 was tyrosine-phosphorylated in Src-activated cancer cells. In addition to 4T1 and AsPC1 cells, SW620 colon cancer cells also expressed hyper-activated Src [53]. SW620 cells were derived from lymph node metastasis of the same cancer patient as SW480 cells [54]. We infected these high-Src cells as well as low-Src SW480 cells

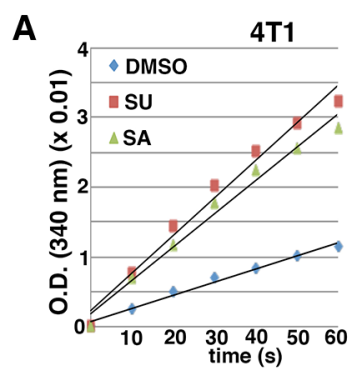

C

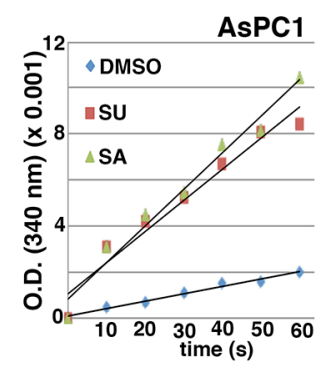

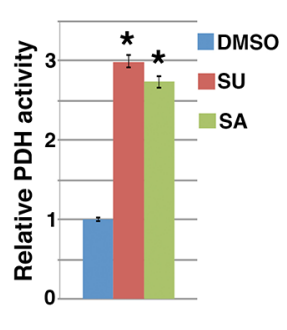

B
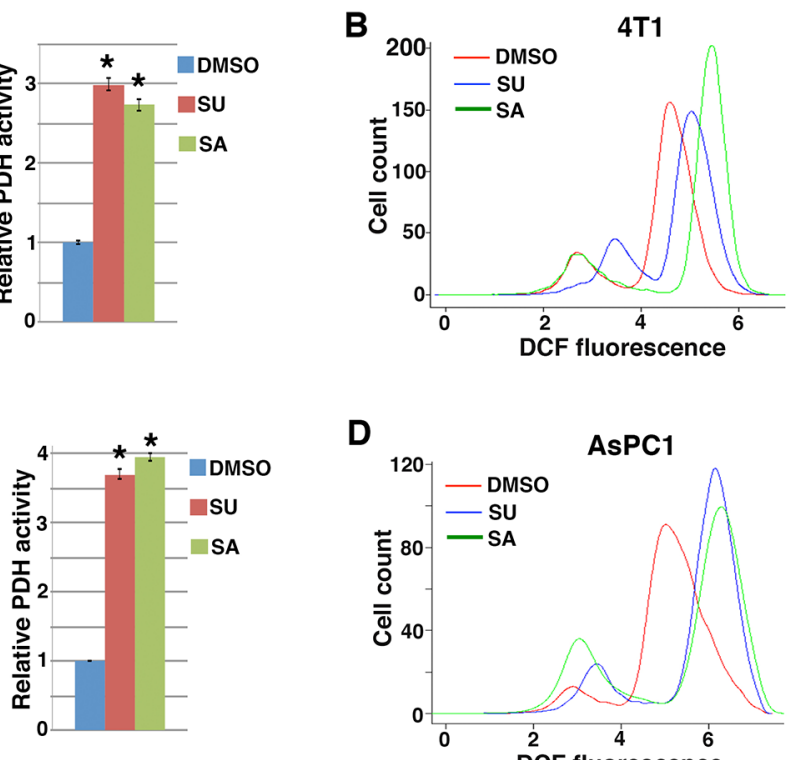

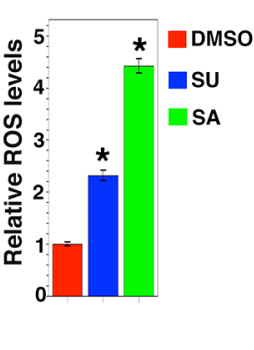

D
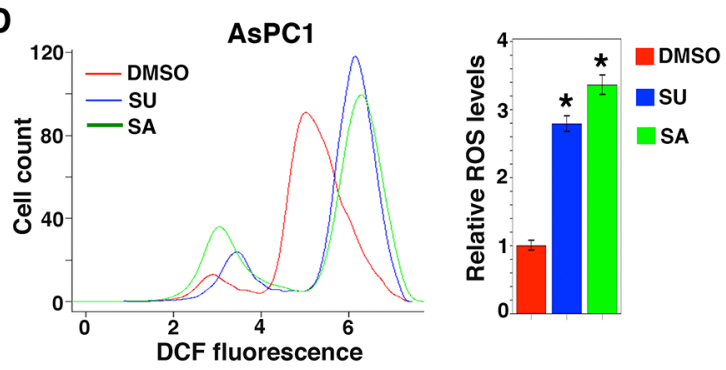

Figure 2: Src inhibitors activate PDH and enhance ROS production. 4T1 A, B. and AsPC1 C, D. cells were treated with indicated Src inhibitors, followed by measurement of PDH activity (A, C). and ROS levels (B, D). SU6656 (SU): $2 \mu$ M, Saracatinib (SA): $2 \mu \mathrm{M}$. Error bars represent S.D. *p $<0.01$. 
(negative control) with lentiviral PDHA1-Flag. PDHA1Flag proteins were immunoprecipitated with anti-Flag antibodies followed by immunoblotting for phosphotyrosine. Tyrosine phosphorylation of PDHA1-Flag was indeed detected in SW620, 4T1 and AsPC1 cells, but not in SW480 cells (Figure 3B), suggesting that PDHA1 tyrosine phosphorylation correlates with Src activity. To further determine whether tyrosine phosphorylation of PDHA1 might require PDK-mediated serine phosphorylation, we stably expressed the AAA mutant form of PDHA1-Flag that lacked the three serine phosphorylation sites for PDKs through lentiviral infection [4]. PDHA1 AAA mutant also exhibited tyrosine phosphorylation in SW620, 4T1 and AsPC1 cells (Figure 3B), suggesting that PDHA1 tyrosine phosphorylation is independent of PDK-mediated serine phosphorylation.

We next asked whether PDHA1 tyrosine phosphorylation in these cancer cells was dependent on Src. We treated PDHA1-Flag-transduced SW620, 4T1 and AsPC1 cells with chemically distinct Src kinase inhibitors,
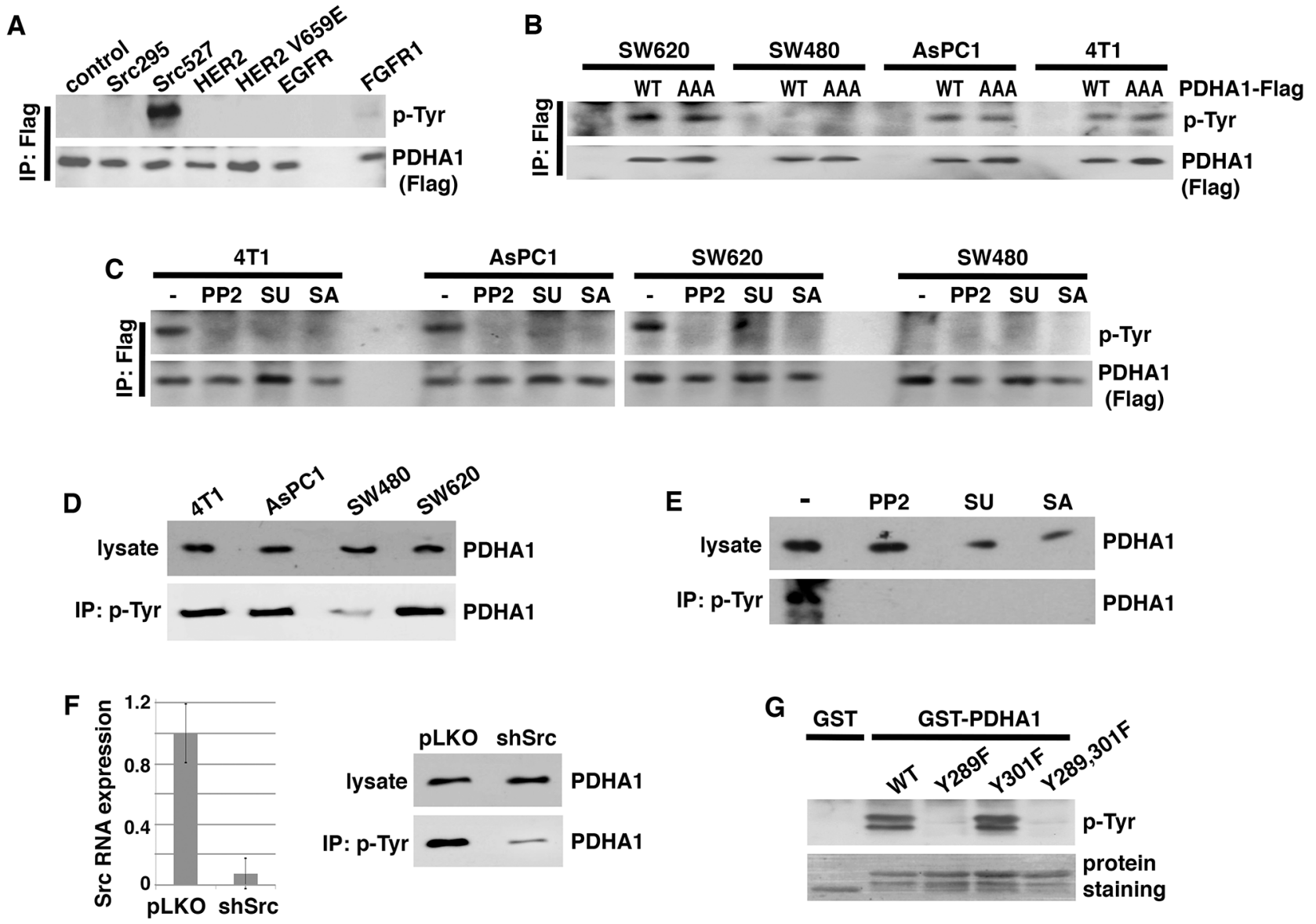

Figure 3: Src is required for PDHA1 tyrosine phosphorylation in cancer cells and directly phosphorylates PDHA1 at Y289. A. Src induces PDHA1 tyrosine phosphorylation. PDHA1 with a carboxyl-terminal Flag tag (PDHA1-Flag) was transiently transfected into HEK293 cells together with indicated tyrosine kinases. PDHA1 was immunoprecipitated with anti-Flag antibodies and immunoblotted with anti-phosphotyrosine (p-Tyr) antibodies. B. Exogenous PDHA1 is tyrosine-phosphorylated in cancer cells. Indicated cancer cells were transduced with lentivirus expressing wild type (WT) or AAA mutant PDHA1-Flag, followed by immunoprecipitation with anti-Flag antibodies and immunoblotting with anti-p-Tyr antibodies. AAA mutant PDHA1 lacks PDK phosphorylation sites. C. Src inhibitors block tyrosine phosphorylation of exogenous PDHA1 in cancer cells. Indicated cancer cells were transduced with lentiviral PDHA1-Flag. Cells were treated with Src inhibitors PP2 $(5 \mu \mathrm{M})$, SU $(2 \mu \mathrm{M})$ or SA $(2 \mu \mathrm{M})$, then harvested for immunoprecipitation with anti-Flag antibodies and immunoblotting for tyrosine phosphorylation. D. Endogenous PDHA1 proteins are tyrosine-phosphorylated in Src-activated cancer cells. Whole cell lysates were prepared from indicated cancer cells, and subjected to either immunoblotting with antiPDHA1 antibodies or immunoprecipitation with anti-p-Tyr antibodies, followed by immunoblotting with anti-PDHA1. E. Src inhibitors abolish tyrosine phosphorylation of endogenous PDHA1. AsPC1 cancer cells were treated with PP2 $(5 \mu \mathrm{M}), \mathrm{SA}(1 \mu \mathrm{M})$ or SU $(1 \mu \mathrm{M})$, followed by immunoprecipitation with anti-p-Tyr antibodies and immunoblotting with anti-PDHA1 antibodies. F. Src is required for tyrosine phosphorylation of endogenous PDHA1. AsPC1 cells were infected with lentiviral vector (pLKO) or shRNA targeting Src (shSrc). Src depletion efficiency was verified by quantitative RT-PCR. Cells were lysed for immunoprecipitation with anti-p-Tyr antibodies, followed by immunoblotting with anti-PDHA1 antibodies. G. Src phosphorylates Y289 of PDHA1. WT and tyrosine mutant forms of PDHA1 (aa 281-356) were fused to GST. The purified fusion proteins were incubated with recombinant Src enzyme, followed by immunoblotting with anti-p-Tyr antibodies. 
including PP2, SU6656, and Saracatinib [37-39], and immunoprecipitated PDHA1-Flag to determine the tyrosine phosphorylation status. All three Src inhibitors abolished PDHA1 tyrosine phosphorylation (Figure 3C). This result suggests that Src kinases are essential for PDHA1 tyrosine phosphorylation in these cancer cells.

Based on the observations of exogenous PDHA1, we sought to determine whether endogenous PDHA1 proteins were tyrosine-phosphorylated by Src. The protein levels of endogenous PDHA1 were comparable among SW620, 4T1, AsPC1 and SW40 cells (Figure 3D). We immunoprecipitated tyrosine-phosphorylated proteins from these cells using anti-phospho-tyrosine antibodies, and examined the protein abundance of PDHA1 in the precipitates. Tyrosine-phosphorylated PDHA1 levels in SW480 cells were much lower than those in SW620, 4T1 and AsPC1 cells (Figure 3D). To validate that endogenous PDHA1 tyrosine phosphorylation in the high-Src cells was attributed to Src, we treated AsPC1 cells with Src inhibitors PP2, SU6656, and Saracatinib. Isolation of tyrosine-phosphorylated proteins from treated cells confirmed that all Src inhibitors abolished tyrosine phosphorylation of endogenous PDHA1 (Figure 3E). Finally, we depleted Src in AsPC1 cells using lentiviral short-hairpin RNA (shRNA) (Figure 3F). Depletion of Src did not change the total protein levels of endogenous PDHA1, however, it strongly decreased the abundance of tyrosine-phosphorylated PDHA1 (Figure 3F). Taken together, these results demonstrate that PDHA1 is tyrosine-phosphorylated in cancer cells in a Src-dependent manner.

\section{Src directly phosphorylates tyrosine 289 of PDHA1}

To investigate whether Src might directly phosphorylate PDHA1, we performed in vitro kinase assay. Large-scale phospho-proteomics studies have revealed that PDHA1 could be phosphorylated at multiple tyrosine (Y) residues in various normal and tumor cells, with Y289 and Y301 as the most heavily phosphorylated tyrosine sites (http://www.phosphosite.org). Y301 was reported to be the FGFR1 phosphorylation site [52]. We prepared bacteriaexpressed recombinant PDHA1 protein that was fused to glutathione S-transferase (GST). We also generated mutant GST-PDHA1 fusion proteins in which Y289 and Y301 of PDHA1 were substituted with phenylalanine (F). The WT and mutant GST-PDHA1 proteins were incubated with recombinant active Src enzyme, followed by immunoblotting with phospho-tyrosine antibodies. Tyrosine phosphorylation of WT and Y301F mutant PDHA1 by Src was readily detected (Figure 3G). However, PDHA1 Y289F mutant completely resisted Src-mediated tyrosine phosphorylation (Figure $3 \mathrm{G}$ ). These results suggest that Src can directly phosphorylate PDHA1 specifically at Y289. Therefore, PDHA1 is a new substrate of Src.
PDHA1 Y289 is a highly conserved residue (Supplementary Figure S2). To understand the consequence of its phosphorylation on PDH activity, we examined the crystal structure of PDH [10]. PDHcatalyzed decarboxylation of pyruvate requires thiamin diphosphate (ThDP or TPP) [9]. In TPP-bound PDHA1 [10], Arginine (R) 288 is one of the critical TPP-anchoring residues, and Y289 is in close proximity to Aspartic acid (D) 315 (Supplementary Figure S3). Y289 is located at the protein surface and is accessible to enzymes that may modify it. Upon phosphorylation, the bulky phosphoryl group at Y289 will pose a steric clash with D315. The resultant repulsion is expected to affect the positioning of Y289 and hence the neighboring R288, thereby interfering with the binding of TPP and the enzymatic activity. This model is consistent with the observation that Src decreased PDH activity.

\section{PDHA1 Y289 phosphorylation is essential for Src's metabolic and pro-malignant effects}

Activated Src can phosphorylate many substrates implicated in a variety of malignant phenotypes [55]. It was unclear whether PDHA1 Y289 phosphorylation by Src might be biologically significant in regards to Srcmediated oncogenic function. We stably expressed the Src-resistant PDHA1 Y289F mutant in Src-activated cancer cells through lentiviral transduction, and examined whether it reversed Src's effect on metabolism and cell proliferation/survival. When expressed in $4 \mathrm{~T} 1$ cells (Figure 4A), both WT and Y289F mutant PDHA1 increased PDH activity, but Y289F mutant exhibited a substantially stronger effect than WT PDHA1 (Figure 4B). This result suggests that Src inhibits PDH in large part through PDHA1 Y289 phosphorylation. Consistent with the PDH activity, 4T1 cells expressing Y289F PDHA1 displayed higher oxygen consumption rates and ROS content than those expressing WT PDHA1 (Figure 4C and 4D). Similarly, in SW620 cells, Y289F PDHA1 also led to more robust PDH activation and ROS generation than WT PDHA1 (Supplementary Figure S4A and Supplementary Figure S4B). These results support that PDHA1 Y289 phosphorylation is essential for Src to suppress PDH activity and mitochondrial oxidative metabolism.

Heightened oxidative stress reduces cell growth and viability $[7,8]$, and sensitizes cancer cells to anoikis as well as suppresses metastasis $[4,5,32,56]$. Expression of WT PDHA1 in 4T1 and SW620 cells did not significantly affect cell growth, however, expression of Y289F PDHA1 decreased cell proliferation (Figure 4E and Supplementary Figure S4C). As Src activation conferred anoikis resistance (Figure 1C), the viability of SW620 cells was not altered when they were cultured in suspension (Supplementary Figure S4D). But increased cell death was observed in cells expressing Y289F PDHA1 compared with cells expressing WT PDHA1 following cell detachment (Supplementary 
Figure S4D). Because metastatic cancer cells need to survive the rigors of the dissemination journey, resistance to anoikis is a prerequisite for metastasis [57]. 4T1 cancer cells are highly metastatic [58]. In the experimental metastasis assay, 4T1 cells expressing PDHA1 Y289F generated fewer lung metastases than cells expressing WT PDHA1 after injection into tail vein of recipient mice (Figure 4F). Collectively, these results suggest that Src's pro-malignant activity is at least in part attributed to its metabolic reprogramming effect through PDHA1 Y289 phosphorylation.

Because Src-mediated PDHA1 tyrosine phosphorylation is independent of PDK-mediated serine phosphorylation, we asked whether simultaneous inhibition of Src and PDK activities might additively induce ROS generation. Dichloroacetate (DCA) is a commonly used PDK inhibitor, acting to activate PDH and oxidative metabolism [59-61]. DCA treatment increased ROS levels in 4T1 cells expressing WT PDHA1 (Figure 5A). Compared with WT PDHA1, expression of Srcresistant Y289F PDHA1 also enhanced ROS production. DCA treatment of cells expressing Y289F PDHA1 led to an additive effect on ROS stimulation (Figure 5A).
The results are consistent with the model that Src and PDK independently suppress PDH and ROS generation (Figure 5B).

\section{Src confers resistance to pro-oxidant therapy through suppression of oxidative metabolism}

Ionizing radiation and many chemotherapy drugs kill cancer cells by directly or indirectly generating free radicals $[6,8]$. Therefore, cancer cells with decreased oxidative stress and/or enhanced antioxidant capacity exhibit intrinsically heightened resistance to pro-oxidants. Because activation of Src reduced $\mathrm{PDH}$ activity and ROS levels (Figure 1 and Supplementary Figure S1), we hypothesized that Src's metabolic effect might contribute to therapeutic resistance.

As Src inhibitors SU6656 and Saracatinib activated PDH and stimulated ROS production (Figure 2), we tested whether they could sensitize Src-activated cancer cells to pro-oxidants. Chemodrug Doxorubicin, a topoisomerase II inhibitor, is able to augment ROS generation [6]. We treated 4T1 cells with Doxorubicin and Src inhibitors singly or in combination, and measured ROS levels and
A

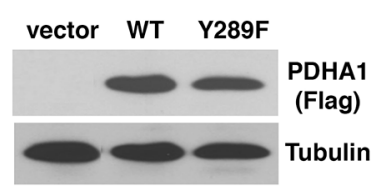

B
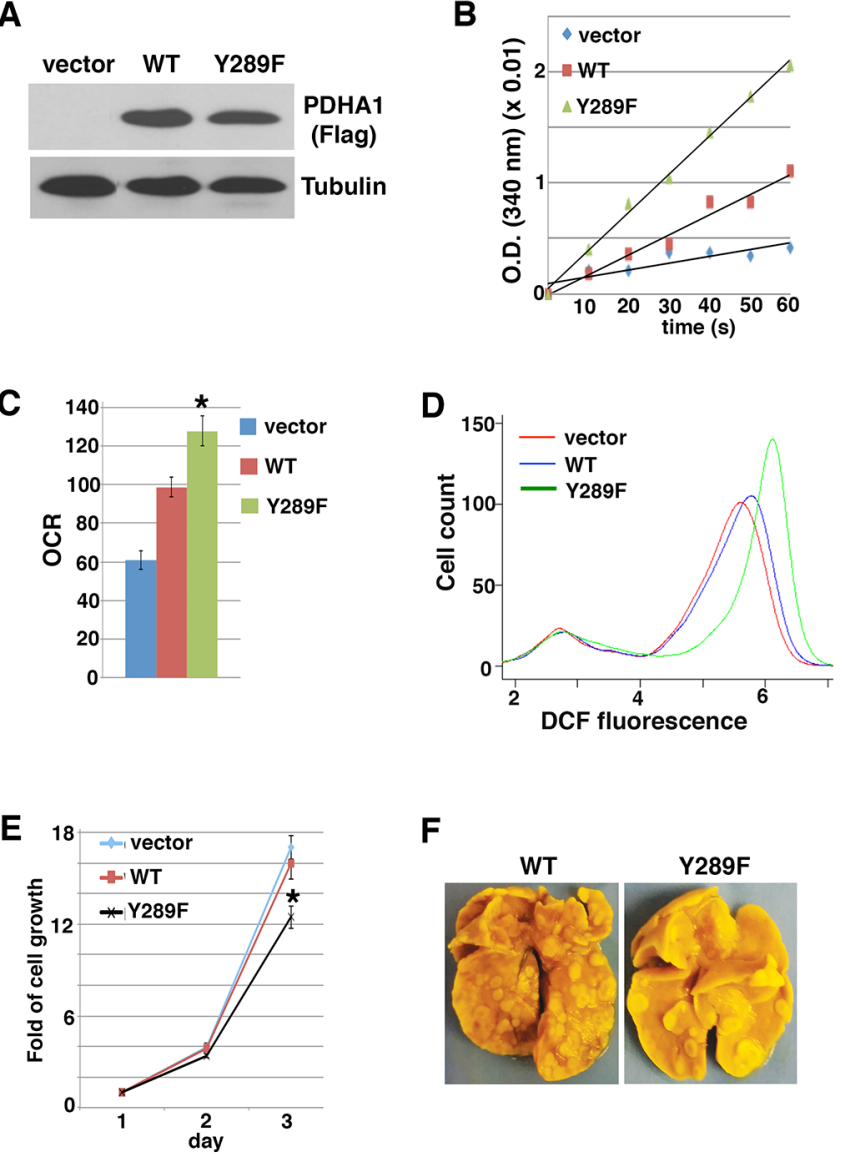

$\mathbf{F}$

C
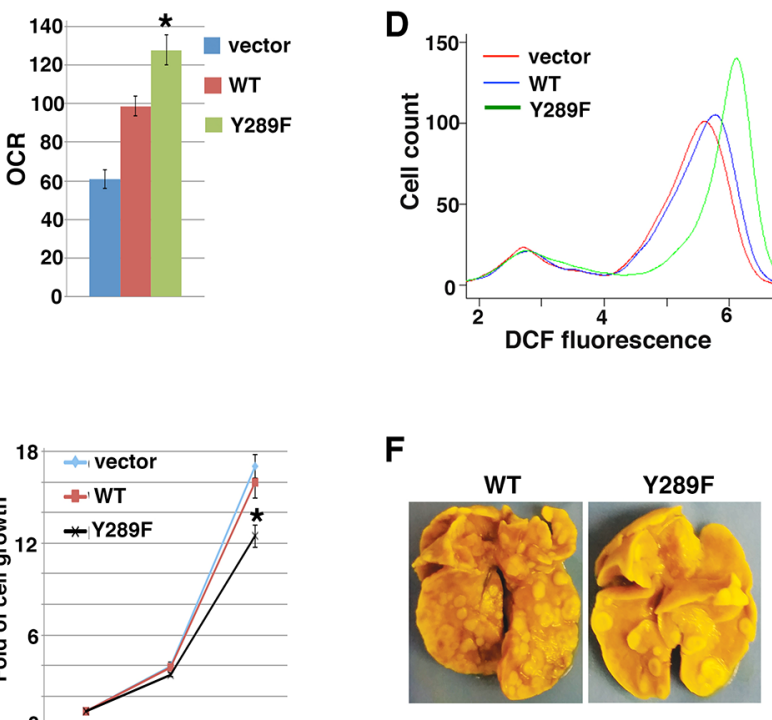
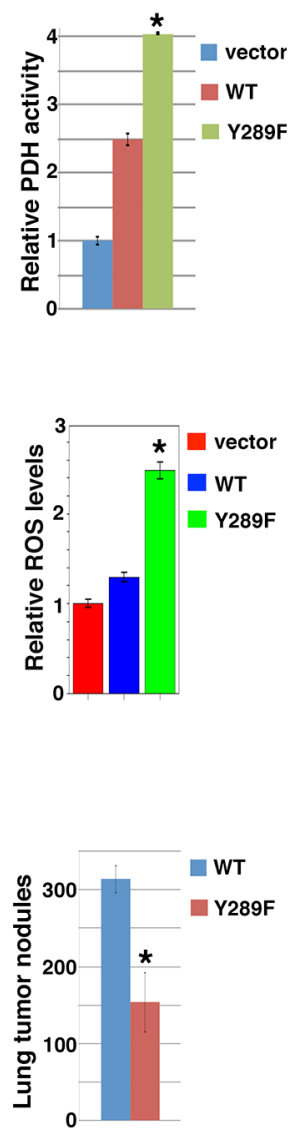

Figure 4: PDHA1 Y289F mutant activates PDH and oxidative metabolism, and reduces cell growth and metastasis. 4T1 cells were transduced with lentiviral control vector or PDHA1-Flag (WT or Y289F), followed by analyses of immunoblotting with antiFlag antibodies A. PDH activity B. oxygen consumption rate (OCR) C. ROS levels D. cell growth (living cell number) E. and metastatic potential in the experimental metastasis assay $(n=5)$ F. Error bars represent S.D. ${ }^{*} p<0.05$. 
cell numbers. Doxorubicin and SU6656 each caused 2-2.5 fold increases in ROS levels, but combination of both drugs led to a nearly 5-fold increase of ROS (Figure 6A). Like SU6656, Saracatinib also exhibited an additive effect on increasing ROS levels when combined with Doxorubicin (Figure 6A). Doxorubicin alone modestly reduced the growth rate and viability of 4T1 cells (Figure

A

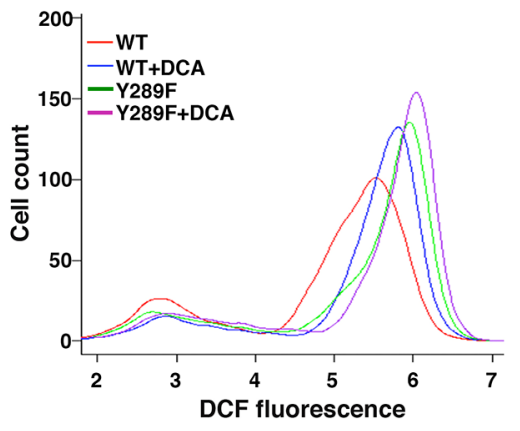

6B). As expected, the Src inhibitors synergized with Doxorubicin to suppress cell growth and survival (Figure 6B). The results show that Src inhibitors can sensitize cancer cells to pro-oxidant chemotherapy.

To verify that Src promoted therapy resistance through its metabolic reprogramming activity, more specifically, PDHA1 Y289 phosphorylation, we compared

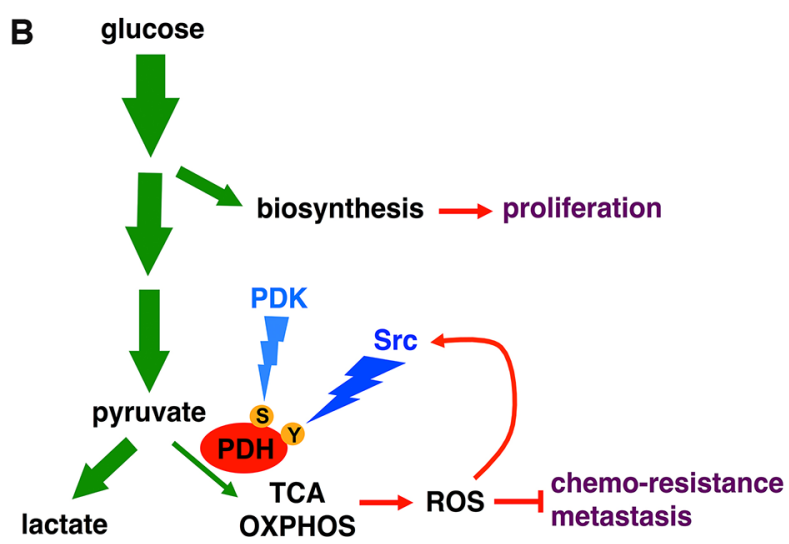

Figure 5: Src and PDK independently suppress ROS production. A. Inhibition of both Src and PDKs additively increases ROS levels. 4T1 cells transduced with WT or Src-resistant Y289F PDHA1 were treated with DCA (10 mM), followed by ROS measurement. Error bars represent S.D. ${ }^{*} p<0.05$. B. A simplified view of glucose metabolic reprogramming in cancer. During glycolysis in cancer cells, a significant portion of glucose carbon is diverted to biosynthetic pathways to fuel cell proliferation. Entry of pyruvate into mitochondrial oxidative metabolism is mediated primarily by the PDH complex. In cancer cells, the great majority of pyruvate is converted to lactate and is kept away from mitochondria, which is in part due to inhibition of PDH. This metabolic feature allows cancer cells to evade overproduction of ROS that are byproducts of mitochondrial oxidative metabolism, thereby acquiring resistance to pro-oxidant therapies and enhanced metastatic potential. Src and PDK independently inactivate PDH through direct tyrosine (Y) and serine (S) phosphorylation, respectively. Furthermore, ROS may activate Src, resulting in a negative feedback loop to restrain cellular oxidative stress (see discussion). Green arrows indicate the flow of glucose carbon. OXPHOS: oxidative phosphorylation.
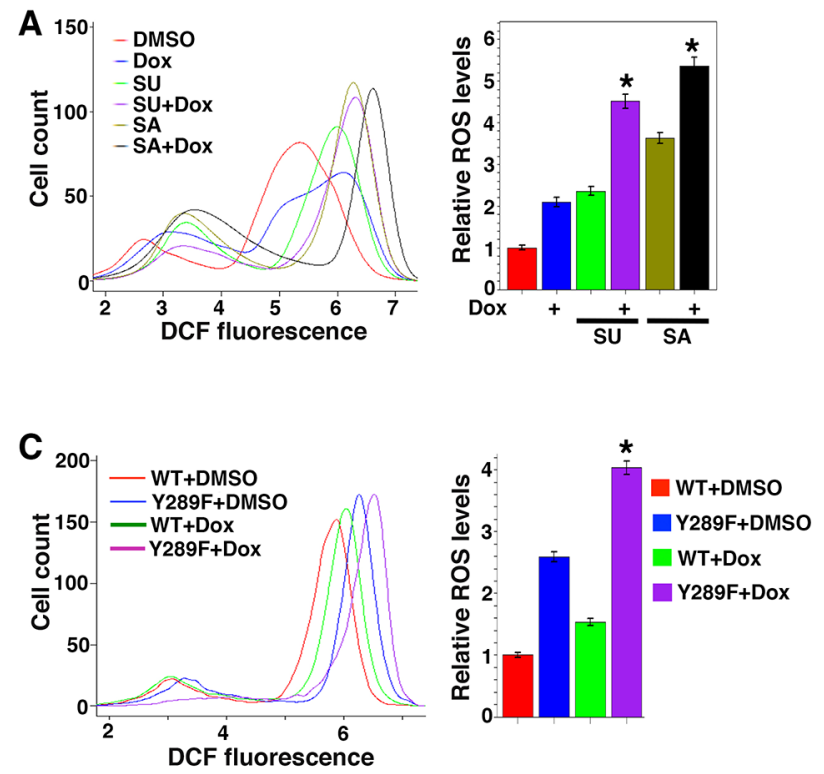
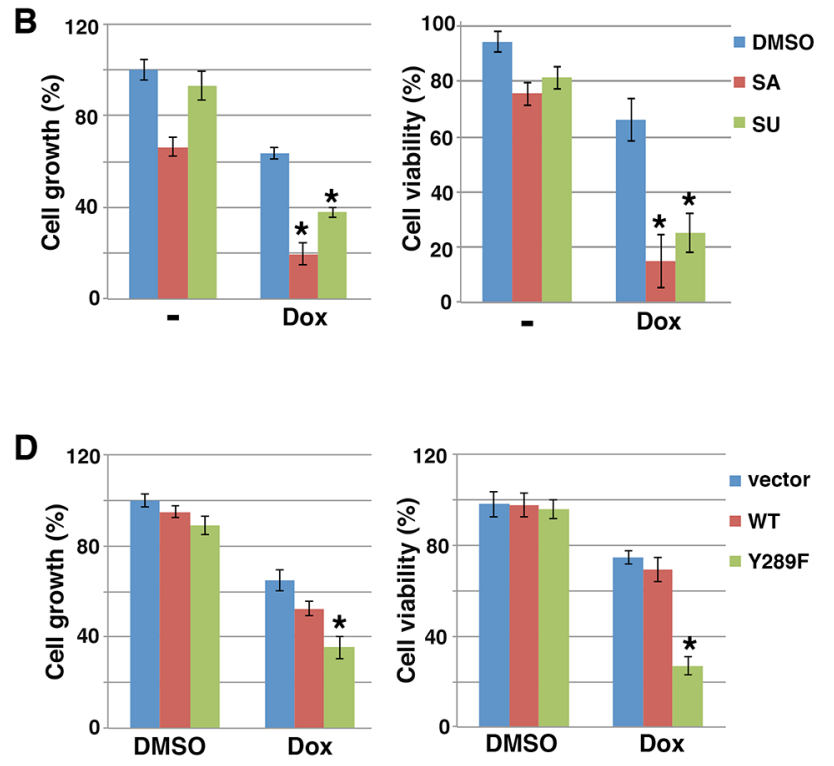

Figure 6: Blocking Src's metabolic effect sensitizes cancer cells to pro-oxidant chemotherapy. A, B. Src inhibitors and chemodrug Doxorubicin (Dox) show additive/synergistic effects on ROS induction and suppression of cell growth/viability. 4T1 cells were treated with Dox $(1 \mu \mathrm{g} / \mathrm{ml})$ and Src inhibitors SA and SU (1 $\mu \mathrm{M}$ each). ROS levels (A) and cell growth and viability (B) were subsequently determined. C, D. Expression of PDHA1 Y289F sensitizes cells to pro-oxidant. 4T1 cells transduced with WT or Y289F PDHA1 were treated with vehicle (DMSO) or Dox, followed by measurement of ROS levels (C) and cell growth and survival (D). Error bars represent S.D. ${ }^{*} \mathrm{p}<0.05$. 
4T1 cells expressing WT or Y289F PDHA1 for their sensitivity to Doxorubicin. Y289F PDHA1-expressing cells indeed generated more ROS than cells expressing WT PDHA1 when both were treated with Doxorubicin (Figure 6C). Accordingly, cells expressing PDHA1 Y289F were markedly more sensitive to Doxorubicin than cells expressing WT PDHA1 with regard to cell growth and survival (Figure 6D). Taken together, these results suggest that Src-mediated metabolic regulation through PDHA1 Y289 phosphorylation promotes cancer cell's resistance to pro-oxidant chemotherapy.

\section{DISCUSSION}

The Warburg effect, which describes the uncoupling of glucose oxidation from increased glycolysis in cancer cells, promotes tumor growth, metastasis, and therapy resistance (Figure 5B) $[2,5,7]$. Src is a classical oncogene with well-established pro-malignant functions. However, the potential role of Src in metabolism and its link to the Warburg effect remained largely elusive. Early studies in 1980s reported that cells infected with viral Src showed signs of increased glucose transport and glycolysis [62, 63]. The present study reveals that Src, which is present in mitochondria in addition to cytoplasm [40-47], can inactivate $\mathrm{PDH}$ through direct tyrosine phosphorylation, thereby attenuating the flux of glycolysis-derived pyruvate into mitochondrial oxidative metabolism. The function of Src in metabolic regulation thus parallels that of PDKs (Figure 5B). Collectively, these findings uncover Src's metabolic reprogramming role and elucidate a biochemical mechanism by which Src contributes to the Warburg phenotype in cancer cells.

Ionizing radiation and many chemotherapy drugs exert cytotoxic activities in large part by directly or indirectly inducing $\operatorname{ROS}[6,8]$. Cancer cells with lower levels of oxidative stress can tolerate higher levels of pro-oxidants $[7,8,64]$. Because Src can inactivate $\mathrm{PDH}$ through tyrosine phosphorylation and attenuate mitochondrial ROS production, Src-activated cancer cells are expected to be intrinsically resistant to prooxidant therapy. This is consistent with the fact that Srcactivated cancers are generally refractory to conventional therapies. Moreover, Src is a redox-sensing kinase and is activated through direct oxidation of its cysteine residues by ROS [65-69]. When cancers with low Src activity are treated with pro-oxidants, Src may become activated by ROS. Indeed, it was reported that chemodrug Oxaliplatin activated Src through induction of intracellular ROS [70]. ROS-activated Src is expected to in turn inhibit PDH to decrease mitochondrial ROS production. This negative feedback may thus maintain cellular redox homeostasis and enable cancer cells to acquire resistance to prooxidants (Figure 5B). Taken together, Src's metabolic effect confers both intrinsic and acquired resistance to pro-oxidant therapy. Combination of Src inhibitors with pro-oxidants may boost ROS generation and lead to synergistic therapeutic effects (Figure 6B).

\section{MATERIALS AND METHODS}

\section{Cell culture, chemicals, antibodies and constructs}

HEK293, SW480, SW620 and 4T1 cells were grown in Dulbecco's modified Eagle's medium (DMEM) supplemented with $10 \%$ bovine calf serum. AsPC1 cells were cultured in RPMI-1640 medium with 10\% fetal bovine serum (FBS). MCF-10A cells were grown in DMEM/F12 with 5\% Horse Serum, 20ng/ml EGF, 0.5 $\mathrm{mg} / \mathrm{ml}$ Hydrocortisone, $10 \mu \mathrm{g} / \mathrm{ml}$ Insulin. Src inhibitors PP2, SU6656 and Saracatinib were purchased from Cayman Chemical, and chemodrug Doxorubicin from Sigma. Mouse and rabbit phospho-tyrosine (P-Tyr-1000 and P-Tyr-100) and PDHA1 monoclonal antibodies were from Cell Signaling. Mouse monoclonal Flag M2 antibody was from Sigma. PDHA1 AAA mutant was created by substituting three serine residues (S293, S300, and S232) with alanine. PDHA1 Y289F mutant was generated by replacing tyrosine 289 with phenylalanine. Mutagenesis was achieved using a method described in QuickChange II Site-Directed Mutagenesis (Stratagene).

\section{Preparation of mitochondrial extract}

Mitochondria were prepared as described [71]. Cells $\left(1 \times 10^{8}\right)$ were washed once with $10 \mathrm{ml}$ Grinding medium (Sucrose 250 mM, EDTA 2 mM, BSA $1 \mathrm{mg} / \mathrm{ml}, \mathrm{pH} 7.4$ ), and centrifuged for $5 \mathrm{~min}$ at $800 \mathrm{~g}$ at $4^{\circ} \mathrm{C}$. The pellet was re-suspended in $1 \mathrm{ml}$ Grinding medium, sonicated, and centrifuged for $12 \mathrm{~min}$ at $800 \mathrm{~g}$ at $4^{\circ} \mathrm{C}$. The supernatant was collected, followed by immediate centrifuge for 20 $\min$ at $8,500 \mathrm{~g}$ at $4^{\circ} \mathrm{C}$. The pellet was re-suspended with $500 u$ buffer S (Sucrose $150 \mathrm{mM}, \mathrm{KCl} 40 \mathrm{mM}$, Tris/ $\mathrm{HCl} 25 \mathrm{mM}$, BSA $1 \mathrm{mg} / \mathrm{ml}, \mathrm{pH} 7.4$ ), sonicated and then centrifuged $20 \mathrm{~min}$ at $10,000 \mathrm{~g}$ at $4^{\circ} \mathrm{C}$. Mitochondrial fractions in the pellet were re-suspended in $100 \mathrm{ml}$ buffer $\mathrm{S}$. Protein concentration in mitochondrial fractions were determined by BCA Protein Assay.

\section{PDH activity assay}

PDH-mediated conversion of pyruvate to acetylCoA generates NADH. PDH activity measurement was based on the rate of NADH generation (as described in [72]). Pyruvate decarboxylase activity was assayed immediately after preparation of the mitochondrial extracts, at $30^{\circ} \mathrm{C}$ with a spectrophotometer set at $340 \mathrm{~nm}$. The assay mixture contained the following: potassium phosphate buffer ( $\mathrm{pH} 8.0), 100 \mathrm{mM}$; $\mathrm{MgCl}$, $1 \mathrm{mM}$; thiamine pyrophosphate, $0.2 \mathrm{mM}$; NAD, 2.5 $\mathrm{mM}$; cysteine- $\mathrm{HCl}, 2 \mathrm{mM}$; pyruvate, $5 \mathrm{mM}$; Triton 
$\mathrm{X}-100,0.05 \%$; and mitochondrial extract. The reaction was started by addition of $0.15 \mathrm{mM}$ coenzyme A. Reaction rates were linearly proportional to the amount of extract added. Each experiment was carried out in triplicate.

\section{In vitro kinase assay}

GST-PDHA1 (WT and mutant) fusion proteins that were conjugated on glutathione beads were incubated with purified Src recombinant enzyme (SignalChem) in the Src assay buffer (Hepes 50mM pH7.5, $\mathrm{MgCl}_{2} 5 \mathrm{mM}$, ATP $50 \mu \mathrm{m}, \mathrm{NaCl} 150 \mathrm{mM}$, DTT $1 \mathrm{mM}$, NP-40 0.02\%) for $30 \mathrm{~min}$ at $30^{\circ} \mathrm{C}$. The GST-beads were washed with co-immunoprecipitation buffer (Tris $20 \mathrm{mM} \mathrm{PH7.5,} \mathrm{NaCl}$ $150 \mathrm{mM}$, NP-40 0.5\%). Western blotting was carried out with Rabbit anti- Phospho-Tyrosine (P-Tyr-1000) monoclonal antibody (Cell Signaling).

\section{Quantification of oxygen consumption rate (OCR) and ROS}

Oxygen consumption rates were determined with a Seahorse XF Analyzer. Cellular ROS levels were evaluated with carboxy-H2DCFDA (5-(and-6)carboxy-2, 7'-dichlorodihydrofluorescein diacetate) from Acros Organics. Oxidation of DCFDA to the highly fluorescent 2,7-dichloro-fluorescein (DCF) is proportionate to ROS generation. Adherent cells were incubated with $5 \mu \mathrm{M}$ DCFDA for 30 minutes in PBS at $37^{\circ} \mathrm{C}$ in the dark. Cells were washed with PBS, trypsinized, rinsed, and resuspended in cold PBS. Fluorescence data were acquired within 60 minutes using the BD ACCURI C6 Flow Cytometer (using the FL1 channel). Analysis of data was conducted by FCS Express 4 Flow and RStudio with package FlowCore. Mean fluorescence intensity was used for comparison. Each experiment was carried out in triplicate and results were presented as means \pm S.D. for each treatment group.

\section{Cell viability, anoikis, and tail vein injection}

Cell viability was determined based on Trypan blue staining using a Bio-Rad TC20 automated cell counter. Anoikis assay was performed as previously reported [4]: MCF10A and SW620 cells expressing WT or Y289F PDHA1 were cultured in suspension for 2 days before counting viable cells. Experimental metastasis assay was conducted by injecting $5 \times 10^{5} 4 \mathrm{~T} 1$ cells into tail vein of NOD/SCID mice (5-6 weeks old) as previously described [4].

\section{ACKNOWLEDGMENTS}

This work was in part supported by NIH R01CA137021 to JL.

\section{CONFLICTS OF INTEREST}

The authors declare no conflict of interest.

\section{REFERENCES}

1. DeBerardinis RJ, Lum JJ, Hatzivassiliou G, Thompson CB. The biology of cancer: metabolic reprogramming fuels cell growth and proliferation. Cell Metabolism. 2008; 7:11-20. doi:10.1016/j.cmet.2007.10.002.

2. Vander Heiden MG, Cantley LC, Thompson CB. Understanding the Warburg effect: the metabolic requirements of cell proliferation. Science. 2009; 324:1029-33. doi:10.1126/science.1160809.

3. Dröse S, Brandt U. Molecular mechanisms of superoxide production by the mitochondrial respiratory chain. Advances in Experimental Medicine and Biology. 2012; 748:145-69. doi:10.1007/978-1-4614-3573-0_6.

4. Kamarajugadda S, Stemboroski L, Nayak S, Simpson NE, Cai Q, Tan M, Lu J. Glucose Oxidation Modulates Anoikis and Tumor Metastasis. Molecular and Cellular Biology. 2012; 32:1893-907. doi:10.1128/MCB.06248-11.

5. Lu J, Tan M, Cai Q. The Warburg effect in tumor progression: Mitochondrial oxidative metabolism as an anti-metastasis mechanism., Cancer Letters. (2014) doi:10.1016/j. canlet.2014.04.001. Epub 2014 Apr.

6. Gorrini C, Harris IS, Mak TW. Modulation of oxidative stress as an anticancer strategy. Nature Reviews. Drug Discovery. 2013; 12:931-47. doi:10.1038/nrd4002.

7. Cairns RA, Harris IS, Mak TW. Regulation of cancer cell metabolism. Nature Reviews. Cancer. 2011; 11:85-95. doi:10.1038/nrc2981.

8. Trachootham D, Alexandre J, Huang P. Targeting cancer cells by ROS-mediated mechanisms: a radical therapeutic approach? Nature Reviews. Drug Discovery. 2009; 8:57991. doi:10.1038/nrd2803.

9. Patel MS, Korotchkina LG. Regulation of the pyruvate dehydrogenase complex. Biochemical Society Transactions. 2006; 34:217-22. doi:10.1042/BST20060217.

10. Kato M, Wynn RM, Chuang JL, Tso S-C, Machius M, Li J, Chuang DT. Structural basis for inactivation of the human pyruvate dehydrogenase complex by phosphorylation: role of disordered phosphorylation loops. Structure. 2008; 16:1849-59. doi:10.1016/j.str.2008.10.010.

11. Kim J, Tchernyshyov I, Semenza GL, Dang CV. HIF-1mediated expression of pyruvate dehydrogenase kinase: a metabolic switch required for cellular adaptation to hypoxia. Cell Metabolism. 2006; 3:177-185. doi:10.1016/j. cmet.2006.02.002.

12. Lu C-W, Lin S-C, Chien C-W, Lin S-C, Lee C-T, Lin B-W, Lee JC, Tsai SJ. Overexpression of pyruvate dehydrogenase kinase 3 increases drug resistance and early recurrence in colon cancer. The American Journal of Pathology. 2011; 179:1405-14. doi:10.1016/j.ajpath.2011.05.050. 
13. Papandreou I, Cairns RA, Fontana L, Lim AL, Denko NC. HIF-1 mediates adaptation to hypoxia by actively downregulating mitochondrial oxygen consumption. Cell Metabolism. 2006; 3:187-197. doi:10.1016/j.cmet.2006.01.012.

14. Pate KT, Stringari C, Sprowl-Tanio S, Wang K, TeSlaa T, Hoverter NP, McQuade MM, Garner C, Digman MA, Teitell MA, Edwards RA, Gratton E, Waterman ML. Wnt signaling directs a metabolic program of glycolysis and angiogenesis in colon cancer. The EMBO Journal. 2014; 33:1454-73. doi:10.15252/embj.201488598.

15. Kim J, Gao P, Liu Y-C, Semenza GL, Dang CV. Hypoxiainducible factor 1 and dysregulated c-Myc cooperatively induce vascular endothelial growth factor and metabolic switches hexokinase 2 and pyruvate dehydrogenase kinase 1. Molecular and Cellular Biology. 2007; 27:738193. doi:10.1128/MCB.00440-07.

16. McFate T, Mohyeldin A, Lu H, Thakar J, Henriques J, Halim ND, Wu H, Schell MJ, Tsang TM, Teahan O, Zhou $\mathrm{S}$, Califano JA, Jeoung NH, et al. Pyruvate dehydrogenase complex activity controls metabolic and malignant phenotype in cancer cells. The Journal of Biological Chemistry. 2008; 283:22700-8. doi:10.1074/jbc.M801765200.

17. Saunier E, Benelli C, Bortoli S. The pyruvate dehydrogenase complex in cancer: An old metabolic gatekeeper regulated by new pathways and pharmacological agents. International Journal of Cancer. 2015. doi:10.1002/ ijc. 29564 .

18. Levine AJ, Puzio-Kuter AM. The control of the metabolic switch in cancers by oncogenes and tumor suppressor genes. Science. 2010; 330:1340-4. doi:10.1126/science.1193494.

19. Boroughs LK, DeBerardinis RJ. Metabolic pathways promoting cancer cell survival and growth. Nature Cell Biology. 2015; 17:351-359. doi:10.1038/ncb3124.

20. Thomas SM, Brugge JS. Cellular functions regulated by Src family kinases. Annual Review of Cell and Developmental Biology. 1997; 13:513-609. doi:10.1146/annurev. cellbio.13.1.513.

21. Yeatman TJ. A renaissance for SRC. Nature Reviews. Cancer. 2004; 4:470-80. doi:10.1038/nrc1366.

22. Martin GS. The hunting of the Src., Nature Reviews. Molecular Cell Biology. 2001; 2:467-75. doi:10.1038/ 35073094.

23. Elsberger B. Translational evidence on the role of Src kinase and activated Src kinase in invasive breast cancer. Critical Reviews in Oncology/hematology. 2014; 89:34351. doi:10.1016/j.critrevonc.2013.12.009.

24. Kim LC, Song L, Haura EB. Src kinases as therapeutic targets for cancer. Nature Reviews. Clinical Oncology. 2009; 6:587-95. doi:10.1038/nrclinonc.2009.129.

25. Zhang S, Yu D. Targeting Src family kinases in anticancer therapies: turning promise into triumph. Trends in Pharmacological Sciences. 2012; 33:122-8. doi:10.1016/j. tips.2011.11.002.

26. Mayer EL, Krop IE. Advances in targeting SRC in the treatment of breast cancer and other solid malignancies. Clinical
Cancer Research. 2010; 16:3526-32. doi:10.1158/10780432.CCR-09-1834.

27. Cooper JA, Gould KL, Cartwright CA, Hunter T. Tyr527 is phosphorylated in pp60c-src: implications for regulation. Science. 1986; 231:1431-4.

28. Kmiecik TE, Shalloway D. Activation and suppression of pp60c-src transforming ability by mutation of its primary sites of tyrosine phosphorylation. Cell. 1987; 49:65-73.

29. Windham TC, Parikh NU, Siwak DR, Summy JM, McConkey DJ, Kraker AJ, Gallick GE. Src activation regulates anoikis in human colon tumor cell lines. Oncogene. 2002; 21:7797-807. doi:10.1038/sj.onc.1205989.

30. Frisch SM, Ruoslahti E. Integrins and anoikis. Current Opinion in Cell Biology. 1997; 9:701-6.

31. Li AE, Ito H, Rovira II, Kim KS, Takeda K, Yu ZY, Ferrans VJ, Finkel T. A role for reactive oxygen species in endothelial cell anoikis. Circulation Research. 1999; 85:304-10.

32. Kamarajugadda S, Cai Q, Chen H, Nayak S, Zhu J, He M, Jin Y, Zhang Y, Ai L, Martin SS, Tan M, Lu J. Manganese superoxide dismutase promotes anoikis resistance and tumor metastasis. Cell Death \& Disease. 2013; 4:e504. doi:10.1038/cddis.2013.20.

33. Schafer ZT, Grassian AR, Song L, Jiang Z, Gerhart-Hines Z, Irie HY, Gao S, Puigserver P, Brugge JS. Antioxidant and oncogene rescue of metabolic defects caused by loss of matrix attachment. Nature. 2009; 461:109-13. doi:10.1038/ nature 08268 .

34. Orrenius S, Gogvadze V, Zhivotovsky B. Mitochondrial oxidative stress: implications for cell death. Annual Review of Pharmacology and Toxicology. 2007; 47:143-83. doi:10.1146/annurev.pharmtox.47.120505.105122.

35. Tanjoni I, Walsh C, Uryu S, A. Tomar A, Nam J-O, Mielgo A, et al. PND-1186 FAK inhibitor selectively promotes tumor cell apoptosis in three-dimensional environments. Cancer Biology \& Therapy. 2010; 9:764-77.

36. Lutz MP, Esser IB, Flossmann-Kast BB, Vogelmann R, Lührs H, Friess H, Büchler MW, Adler G. Overexpression and activation of the tyrosine kinase Src in human pancreatic carcinoma. Biochemical and Biophysical Research Communications. 1998; 243:503-8. doi:10.1006/ bbrc.1997.8043.

37. Hanke JH, Gardner JP, Dow RL, Changelian PS, Brissette WH, Weringer EJ. Discovery of a novel, potent, and Src family-selective tyrosine kinase inhibitor. Study of Lckand FynT-dependent T cell activation., The Journal of Biological Chemistry. 1996; 271:695-701.

38. Blake RA, Broome MA, Liu X, Wu J, Gishizky M, Sun L, Courtneidge SA. SU6656, a selective src family kinase inhibitor, used to probe growth factor signaling. Molecular and Cellular Biology. 2000; 20:9018-27.

39. Hennequin LF, Allen J, Breed J, Curwen J, Fennell M, Green TP, Morgentin R, Norman RA, Olivier A, Otterbein L, Plé PA, Warin N, et al. N-(5-chloro-1,3-benzodioxol-4yl)-7-[2-(4-methylpiperazin-1-yl)ethoxy]-5- (tetrahydro2H-pyran-4-yloxy)quinazolin-4-amine, a novel, highly 
selective, orally available, dual-specific c-Src/Abl kinase inhibitor. Journal of Medicinal Chemistry. 2006; 49:646588. doi:10.1021/jm060434q.

40. Salvi M, Brunati AM, Bordin L, La Rocca N, Clari G, Toninello A. Characterization and location of Srcdependent tyrosine phosphorylation in rat brain mitochondria. Biochimica et Biophysica Acta. 2002; 1589:181-95.

41. Miyazaki T, Neff L, Tanaka S, Horne WC, R. Baron R. Regulation of cytochrome c oxidase activity by c-Src in osteoclasts. The Journal of Cell Biology. 2003; 160:709-18. doi:10.1083/jcb.200209098.

42. Demory ML, Boerner JL, Davidson R, Faust W, Miyake T, Lee I, Hüttemann M, Douglas R, Haddad G, Parsons SJ. Epidermal growth factor receptor translocation to the mitochondria: regulation and effect. The Journal of Biological Chemistry. 2009; 284:36592-604. doi:10.1074/ jbc.M109.000760.

43. Livigni A, Scorziello A, Agnese S, Adornetto A, Carlucci A, Garbi C, Castaldo I, Annunziato L, Avvedimento EV, Feliciello A. Mitochondrial AKAP121 links cAMP and src signaling to oxidative metabolism. Molecular Biology of the Cell. 2006; 17:263-71. doi:10.1091/mbc.E05-09-0827.

44. Itoh S, Lemay S, Osawa M, Che W, Duan Y, Tompkins A, Brookes PS, Sheu SS, Abe J. Mitochondrial Dok-4 recruits Src kinase and regulates NF-kappaB activation in endothelial cells. The Journal of Biological Chemistry. 2005; 280:26383-96. doi:10.1074/jbc.M410262200.

45. Arachiche A, Augereau O, Decossas M, Pertuiset C, Gontier E, Letellier T, Dachary-Prigent J. Localization of PTP-1B, SHP-2, and Src exclusively in rat brain mitochondria and functional consequences. The Journal of Biological Chemistry. 2008; 283:24406-11. doi:10.1074/ jbc.M709217200.

46. Tibaldi E, Brunati AM, Massimino ML, Stringaro A, Colone M, Agostinelli E, Arancia G, Toninello A. SrcTyrosine kinases are major agents in mitochondrial tyrosine phosphorylation. Journal of Cellular Biochemistry. 2008; 104:840-9. doi:10.1002/jcb.21670.

47. Gringeri E, Carraro A, Tibaldi E, D'Amico FE, Mancon M, Toninello A, Pagano MA, Vio C, Cillo U, Brunati AM. Lyn-mediated mitochondrial tyrosine phosphorylation is required to preserve mitochondrial integrity in early liver regeneration. The Biochemical Journal. 2010; 425:401-12. doi:10.1042/BJ20090902.

48. Bagrodia S, Chackalaparampil I, Kmiecik TE, Shalloway D. Altered tyrosine 527 phosphorylation and mitotic activation of p60c-src. Nature. 1991; 349:172-5. doi:10.1038/349172a0.

49. Ding Y, Liu Z, Desai S, Zhao Y, Liu H, Pannell LK, Yi H, Wright ER, Owen LB, Dean-Colomb W, Fodstad O, Lu J, LeDoux SP, et al. Receptor tyrosine kinase ErbB2 translocates into mitochondria and regulates cellular metabolism. Nature Communications. 2012; 3:1271. doi:10.1038/ncomms2236.

50. Hitosugi T, Fan J, Chung T-W, Lythgoe K, Wang X, Xie J, Ge Q, Gu TL, Polakiewicz RD, Roesel JL, Chen GZ,
Boggon TJ, Lonial S, et al. Tyrosine phosphorylation of mitochondrial pyruvate dehydrogenase kinase 1 is important for cancer metabolism. Molecular Cell. 2011; 44:86477. doi:10.1016/j.molcel.2011.10.015.

51. Bargmann CI, Hung MC, Weinberg RA. Multiple independent activations of the neu oncogene by a point mutation altering the transmembrane domain of p185. Cell. 1986; 45:649-57.

52. Fan J, Kang H-B, Shan C, Elf S, Lin R, Xie J, Gu TL, Aguiar M, Lonning S, Chung TW, Arellano M, Khoury HJ, Shin DM, et al. Tyr-301 phosphorylation inhibits pyruvate dehydrogenase by blocking substrate binding and promotes the Warburg effect. The Journal of Biological Chemistry. 2014; 289:26533-41. doi:10.1074/jbc.M114.593970.

53. Emaduddin M, Bicknell DC, Bodmer WF, Feller SM. Cell growth, global phosphotyrosine elevation, and c-Met phosphorylation through Src family kinases in colorectal cancer cells. Proceedings of the National Academy of Sciences of the United States of America. 2008; 105:2358-62. doi:10.1073/pnas.0712176105.

54. Leibovitz A, Stinson JC, McCombs WB, McCoy CE, Mazur KC, Mabry ND. Classification of human colorectal adenocarcinoma cell lines. Cancer Research. 1976; 36:4562-9.

55. Reynolds AB, Kanner SB, Bouton AH, Schaller MD, Weed SA, Flynn DC, Parsons JT. SRChing for the substrates of Src, Oncogene. 2013; 33:4537-4547. doi:10.1038/ onc.2013.416.

56. Piskounova E, Agathocleous M, Murphy MM, Hu Z, Huddlestun SE, Zhao Z, Leitch AM, Johnson TM, DeBerardinis RJ, Morrison SJ. Oxidative stress inhibits distant metastasis by human melanoma cells. Nature. 2015; 527:186-91. doi:10.1038/nature15726.

57. Geiger TR, Peeper DS. The neurotrophic receptor TrkB in anoikis resistance and metastasis: a perspective. Cancer Research. 2005; 65:7033-6. doi:10.1158/00085472.CAN-05-0709.

58. Aslakson CJ, Miller FR. Selective events in the metastatic process defined by analysis of the sequential dissemination of subpopulations of a mouse mammary tumor. Cancer Research. 1992; 52:1399-405.

59. Kankotia S, Stacpoole PW. Dichloroacetate and cancer: New home for an orphan drug? Biochimica et Biophysica Acta. 2014; 1846:617-29. doi:10.1016/j.bbcan.2014.08.005.

60. Bonnet S, Archer SL, Allalunis-Turner J, Haromy A, Beaulieu C, Thompson R, Lee CT, Lopaschuk GD, Puttagunta L, Bonnet S, Harry G, Hashimoto K, Porter CJ, et al. A mitochondria- $\mathrm{K}+$ channel axis is suppressed in cancer and its normalization promotes apoptosis and inhibits cancer growth. Cancer Cell. 2007; 11:37-51. doi:10.1016/j.ccr.2006.10.020.

61. Cairns RA, Papandreou I, Sutphin PD, Denko NC. Metabolic targeting of hypoxia and HIF1 in solid tumors can enhance cytotoxic chemotherapy. Proceedings of the National Academy of Sciences of the United States of America. 2007; 104:9445-50. doi:10.1073/pnas.0611662104. 
62. L. Bosca, M. Mojena, J. Ghysdael, G.G. Rousseau, L. Hue, Expression of the $\mathrm{v}$-src or $\mathrm{v}$-fps oncogene increases fructose 2,6-bisphosphate in chick-embryo fibroblasts. Novel mechanism for the stimulation of glycolysis by retroviruses. The Biochemical Journal. 1986; 236:595-9.

63. Flier JS, Mueckler MM, Usher P, Lodish HF. Elevated levels of glucose transport and transporter messenger RNA are induced by ras or src oncogenes. Science. 1987; 235:1492-5.

64. Nogueira V, Hay N. Molecular pathways: reactive oxygen species homeostasis in cancer cells and implications for cancer therapy. Clinical Cancer Research. 2013; 19:430914. doi:10.1158/1078-0432.CCR-12-1424.

65. Akhand AA, Pu M, Senga T, Kato M, Suzuki H, Miyata T, Hamaguchi M, Nakashima I. Nitric oxide controls sre kinase activity through a sulfhydryl group modification-mediated Tyr-527-independent and Tyr-416-linked mechanism. The Journal of Biological Chemistry. 1999; 274:25821-6.

66. Giannoni E, Buricchi F, Raugei G, Ramponi G, Chiarugi P. Intracellular reactive oxygen species activate Src tyrosine kinase during cell adhesion and anchorage-dependent cell growth. Molecular and Cellular Biology. 2005; 25:6391403. doi:10.1128/MCB.25.15.6391-6403.2005.

67. Yoo SK, Starnes TW, Deng Q, Huttenlocher A. Lyn is a redox sensor that mediates leukocyte wound attraction in vivo. Nature. 2011; 480:109-12. doi:10.1038/nature10632.
68. Acín-Pérez R, Carrascoso I, Baixauli F, Roche-Molina M, Latorre-Pellicer A, Fernández-Silva P, Mittelbrunn M, Sanchez-Madrid F, Pérez-Martos A, Lowell CA, Manfredi G, Enríquez JA. ROS-triggered phosphorylation of complex II by Fgr kinase regulates cellular adaptation to fuel use. Cell Metabolism. 2014; 19:1020-33. doi:10.1016/j. cmet.2014.04.015.

69. Corcoran A, Cotter TG. Redox regulation of protein kinases. The FEBS Journal. 2013; 280:1944-65. doi:10.1111/febs.12224.

70. Kopetz S, Lesslie DP, Dallas NA, Park SI, Johnson M, Parikh NU, Kim MP, Abbruzzese JL, Ellis LM, Chandra J, Gallick GE. Synergistic activity of the SRC family kinase inhibitor dasatinib and oxaliplatin in colon carcinoma cells is mediated by oxidative stress. Cancer Research. 2009; 69:3842-9. doi:10.1158/0008-5472.CAN-08-2246.

71. Abou-Khalil S, Abou-Khalil WH. Pyruvate-dependent oxidative phosphorylation in erythroid and myeloid tumor mitochondria. Archives of Biochemistry and Biophysics. 1985; 236:792-6.

72. Schwab MA, Kölker S, van den Heuvel LP, Sauer S, Wolf NI, Rating D, Hoffmann GF, Smeitink JA, Okun JG. Optimized spectrophotometric assay for the completely activated pyruvate dehydrogenase complex in fibroblasts. Clinical Chemistry. 2005; 51:151-60. doi:10.1373/ clinchem.2004.033852. 